\title{
Understanding the Energetics of Helical Peptide Orientation in Membranes
}

\author{
Durba Sengupta, ${ }^{1}$ Lars Meinhold, ${ }^{1}$ Dieter Langosch, ${ }^{2}$ G. Matthias Ullmann, ${ }^{1,3 *}$ and Jeremy C. Smith ${ }^{1 *}$ \\ ${ }^{1}$ IWR-Computational Molecular Biophysics, University of Heidelberg, Heidelberg, Germany \\ ${ }^{2}$ Dept. für Biowissenschaftliche, Grundlagen, Technische Universität München-Weihenstephan, Freising, Germany \\ ${ }^{3}$ Structural Biology/Bioinformatics, University of Bayreuth, Bayreuth, Germany
}

\begin{abstract}
Understanding the energetic factors determining the positioning and orientation of single-helical peptides in membranes is of fundamental interest in structural biology. Here, a simple 5-slab continuum dielectric model for the membrane is examined that distinguishes between the solvent, headgroup, and core regions. An analytical solution for the electrostatic solvation of a single dipole and an all-atom model of $N$-methylacetamide are used to demonstrate the effect of the dielectric boundaries in the system on peptide dipole orientation. The dipole orientation energy is shown to dominate the electrostatic solvation energy of a polyalanine helix in the membrane. With an additional surface-area-dependent term to account for the cavity formation in the aqueous region, the continuum electrostatics description is used to examine several helical peptides, the atoms of which are explicitly represented with a molecular mechanics force field. The experimentally determined tilt angles of a number of peptides of alternating alanine and leucine residues, and of glycophorin and melittin, are accurately reproduced by the model. The factors determining the tilt angles and their fluctuations are analyzed. The tilt angles of the simpler peptides are found to increase approximately linearly with peptide length; this effect is also rationalized. The analysis and model presented here provide a step toward the prediction of helical membrane protein structure. Proteins 2005; 58:913-922. ๑ 2005 Wiley-Liss, Inc.
\end{abstract}

Key words: membrane helices; polyalanine; glycophorin; melittin; WALP; tilt angle fluctuation; dipole; Poisson-Boltzmann equation

\section{INTRODUCTION}

Membrane proteins constitute about one-third of the human genome and play diverse biological roles in processes such as transport of ions and polar compounds, $\mathrm{pH}$ regulation, signal transduction, and vesicle fusion. ${ }^{1-4}$ They also represent approximately $60 \%$ of drug targets. Structures of several membrane-spanning proteins have been obtained experimentally. ${ }^{2,3}$ These have revealed both $\alpha$ helices and $\beta$ sheets, with the former being more common.
Single-helical peptides have been found to insert into lipid membranes. ${ }^{2,5}$ Understanding the physical effects determining the position and orientation of these singletransmembrane helices is of fundamental interest in the elucidation of the driving forces behind membrane protein structure. Added interest arises from evidence that during membrane protein folding, helix packing may follow helix folding. ${ }^{1,6,7}$

Structural information on membrane helices has been determined experimentally using spectroscopy and X-ray diffraction. $^{8-11}$ The orientations of membrane helices can be roughly divided into 2 classes: transmembrane (e.g., glycophorin $^{1}$ ) and surface aligned (e.g., melittin ${ }^{9}$ ), with, in the latter case, the helix axis being more parallel to the plane of membrane. One of the most studied transmembrane segments is glycophorin, in which the monomers have been shown to tilt $\sim 20^{\circ}$ with respect to the membrane normal. ${ }^{12}$ In contrast, melittin monomers are surface aligned, with the helical axis at the depth of the phospholipid glycerol groups. ${ }^{9}$ The orientations of a number of model synthetic peptides, such as polyalanine, ${ }^{13}$ polyleucine, ${ }^{14}$ and $\mathrm{WALP}^{15-18}$ and $\mathrm{KALP}^{18,19}$ peptides, have also been determined (WALP and KALP are peptides of alternating alanine and leucine residues with flanking tryptophan and lysine residues, respectively). A systematic increase of the tilt angle (i.e., the angle between the helix axis and the bilayer normal) of WALP and KALP peptides has been observed with increase in length, ${ }^{14,16}$ with peptides shorter than the bilayer thickness being roughly parallel to the membrane normal and the longer peptides being appreciably tilted.

The existence of the above experimental data paves the way toward a theoretical understanding of how peptides interact with the heterogeneous solvated membrane environment. Theoretical work on membrane proteins has been performed treating the bilayer atoms and surrounding solvent explicitly or implicitly. Calculations with ex-

Grant sponsor: DFG, Emmy-Noether; Grant number: UL 174/2-1,2. Grant sponsor: Daimler-Benz, Siftung; Grant number: Pr. 02-15/01.

*Correspondence to: Jeremy C. Smith and G. Matthias Ullmann, IWR-Computational Molecular Biophysics, University of Heidelberg, Im Neuenheimer Feld 368, 69120 Heidelberg, Germany. E-mail: biocomputing@iwr.uni-heidelberg.de and matthias.ullmann@unibayreuth.de

Received 20 May 2004; Accepted 21 September 2004

Published online 18 January 2005 in Wiley InterScience (www.interscience.wiley.com). DOI: 10.1002/prot.20383 
plicit lipid bilayer atoms using the molecular dynamics (MD) technique have been performed on several peptides. ${ }^{20-27}$ Although these have furnished a rich variety of biophysical information, the use of an explicit-atom membrane environment is computationally expensive and limits the configurational space that can be explored.

Alternatively, the lipid and solvent molecules can be represented implicitly using continuum models. In the implicit models applied so far, the membrane has been represented as a slab of low dielectric constant embedded in a high dielectric aqueous solvent. Using the PoissonBoltzmann equation to calculate the electrostatic energy, this three-slab model has been applied to estimate the free energy of insertion of alamethicin ${ }^{28}$ and oligoalanine ${ }^{29}$ and the orientation of melittin, ${ }^{21} \mathrm{Src}^{30}$ and the FYVE membrane domains. ${ }^{31}$ Recent developments using the generalized Born (GB) approximation ${ }^{32}$ have made it possible to use the GB approach to calculate solvation energies in a biomembrane. A 3-slab model has been used to calculate the orientation of various single peptides such as melittin, ${ }^{33}$ influenza virus fusion peptide, ${ }^{34}$ and bacteriorhodopsin helical fragments. ${ }^{34}$

The above continuum studies capture certain features of the membrane, such as the hydrophobic nature of the membrane core and a hydrophobic-hydrophilic dielectric boundary, and have successfully accounted for some experimental data. However, a drawback of representing the membrane as a single slab of low dielectric constant is that, in assuming that any location in the membrane is described adequately as either bulk hydrocarbon or bulk water, the headgroup energetics are not adequately modeled. ${ }^{35}$ One consequence of this inadequacy is that the preference of certain residues such as tryptophan and lysine for the headgroup region is absent.

Other models have also been proposed. A semi-implicit model, ${ }^{35}$ in which the membrane-water system is modeled as a lattice of dipoles with varying dipole moments, has been able to reproduce the position of a 20-mer WALP peptide and alamethicin in the membrane. In another study, an implicit solvent description was used to calculate the effective solvation free energy in a membrane environment and correctly distinguished between the transmembrane and surface-aligned orientations of glycophorin and melittin, respectively. ${ }^{36}$ However, neither of these studies attempted to decompose the different contributions to the membrane-peptide interaction or to calculate the characteristic tilt angles of the helix axis.

Here we investigate the energetics of the orientation of membrane-bound $\alpha$ helices by calculating the solvation energy of the peptide in a membrane system (i.e., the free energy for transferring the helix from vacuum into a given position and orientation in the membrane). The headgroup region of the membrane is represented by a slab with a dielectric constant intermediate between that of the bulk solvent and the inner membrane, thus leading to the membrane environment being represented by the 5-slab model shown in Figure 1. The solvation energy is calculated using an all-atom model for the peptide, together with the Poisson-Boltzmann equation for the electrostatic

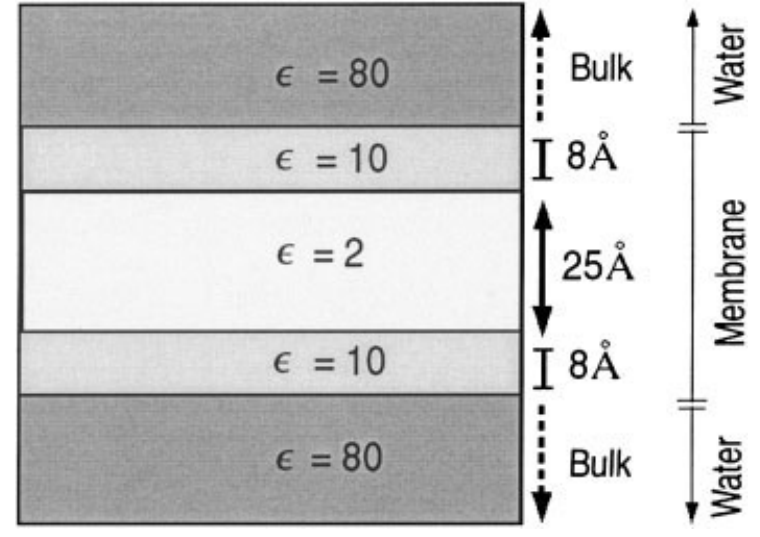

Fig. 1. Five-slab continuum electrostatic model of a biological membrane environment. The membrane is represented as 3 slabs corresponding to the 2 headgroup regions and core region. The headgroup region is modeled as an $8 \AA$ slab $(\varepsilon=10)$ on either side of the low dielectric core region $(\varepsilon=2)$. The 2 outer slabs correspond to bulk water, with $\varepsilon=80$.

solvation energy and a term representing the cost of cavity formation in the aqueous solvent. The most probable orientation is determined by the minimum of the solvation free energy of the peptide in the membrane environment.

The 5-slab model is used to examine the factors determining the position and orientation of single-membrane helices. The energetics of the position and orientation of dipole in a membrane are examined analytically and shown to be a good approximation to the energetics of $N$-methylacetamide, which contains a single peptide group. The peptide dipole energetics are found to tend to orient the axes of single helices perpendicular to the membrane plane, and the calculations indicate that this effect dominates the positioning of polyalanine helices. The length dependence of helix tilt angles and fluctuations in the tilt angles are examined. Cavity formation in the aqueous solvent is also found to play an important role in determining tilt angles of helices longer than the membrane width.

The model reproduces the experimentally determined tilt angles of several membrane-spanning helices, including glycophorin, melittin, and the WALP peptides. Potential uses of the model in the analysis of peptide-membrane interactions and membrane-protein structure prediction are discussed.

\section{THEORY}

The most probable orientation of a membrane helix is that with the lowest solvation free energy, $\Delta G_{\text {solv }}$, defined as the free energy change for transferring the molecule from vacuum to solvent ${ }^{37}$ (the solvent here is the membrane plus the aqueous environment). For a peptide, the position and orientation of a membrane helix are described by 2 parameters: the tilt angle, $\theta$, and the membrane insertion, $v$ (Fig. 2). $\theta$ is the angle between the helix axis and the membrane normal. $v$ is the distance of the center of mass of the helix from the centre of the membrane. Details of the model and the method of calculation of $\Delta G_{\text {solv }}$ are now given. 


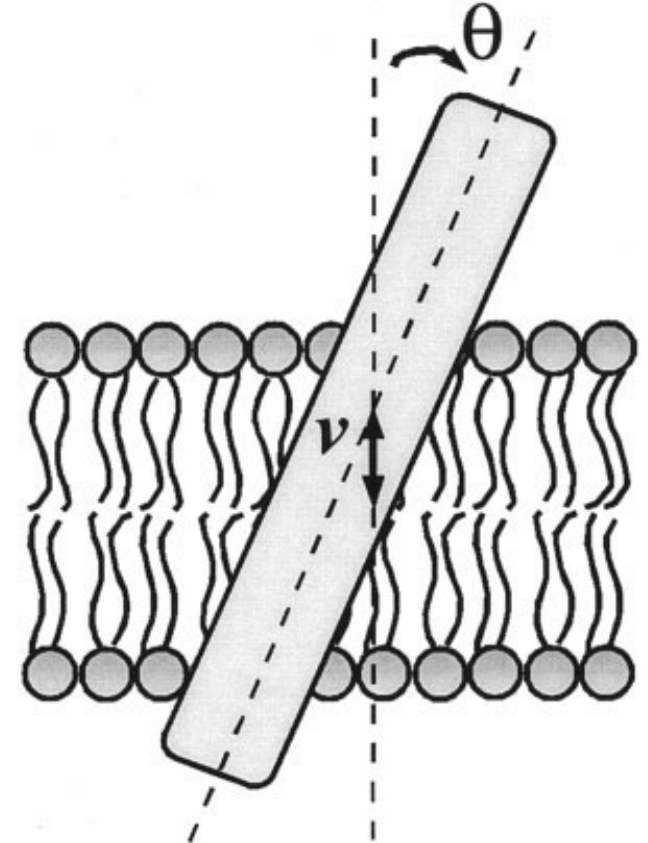

Fig. 2. Parameters describing the orientation of a single helix in the membrane: tilt angle, $\theta$, and extent of membrane insertion, $v$. $\theta$ is the angle between the membrane normal and the helix axis, and $v$ is the distance between the center of the membrane and the center of mass of the helix.

\section{Model}

The model consists of the atomic-detail peptide represented using the CHARMM molecular mechanics poten$\mathrm{tial}^{38}$ in the 5 -slab membrane model shown in Figure 1. The outermost slabs representing the aqueous phase are assigned a dielectric constant of 80 , corresponding to bulk water. A membrane core consists of nonpolar long-chain fatty acids. ${ }^{39,40}$ Correspondingly, the relative dielectric constant of the nonpolar innermost slab is set to $2 .{ }^{39,41}$ The headgroup region in biological membranes is heterogeneous with varying fractions of phosphatidylethanolamine, phosphatidylserine, phosphatidylcholine, sphingomyelin, glycolipids, and cholesterol. Estimates of the dielectric constant of the headgroup region have yielded varying results depending in part on the lipid moieties. ${ }^{39-44}$ The polarity of the headgroup region in phosphatidylcholine and phosphatidylserine bilayers has been found to be between that of bulk water and the bilayer core. ${ }^{43,44}$ In the present case, a value of 10 was used for the dielectric constant in the headgroup region, as models with this value have been found to reproduce the experimental adsorption of small molecules in the head group region. ${ }^{42}$ Variation of this value within reason was found to not significantly affect the results.

\section{Calculation of Solvation Free Energy}

$\Delta G_{\text {solv }}$ was considered to be the sum of 2 contributions:

$$
\Delta G_{\text {solv }}=\Delta G_{\text {elec }}+\Delta G_{n p}
$$

where, $\Delta G_{\text {elec }}$ is electrostatic and $\Delta G_{n p}$ is nonpolar. $\Delta G_{\text {solv }}$ was calculated according to the thermodynamic cycle

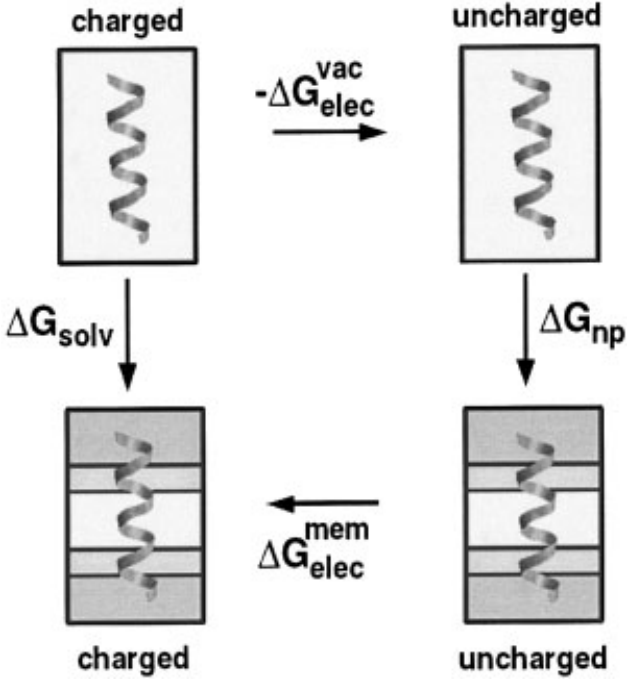

Fig. 3. Four-step thermodynamic cycle for calculating $\Delta G_{\text {solv }}$ for a

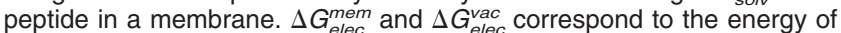
charging the peptide in the membrane and in vacuum, respectively. The nonpolar contribution, $\Delta G_{n p}$, is the cost of cavity formation in the surrounding solvent.

shown in Figure 3. $\Delta G_{\text {elec }}$ is the electrostatic energy required to transfer the peptide from vacuum to a given position in the membrane. It is given by the difference of the energy required to charge the peptide in the membrane, $\Delta G_{\text {elec }}^{\text {mem }}$ and in vacuum, $\Delta G_{\text {elec }}^{\text {vac }}$, that is,

$$
\Delta G_{\text {elec }}=\Delta G_{\text {elec }}^{\text {mem }}-\Delta G_{\text {elec }}^{\text {vac }} \text {. }
$$

$\Delta G_{\text {elec }}$ was calculated using the linearized PoissonBoltzmann equation, which relates the variation in the potential $\theta$ with the spatially dependent dielectric permittivity $\varepsilon(r)$ and the charge distribution $\rho(r)$, and is written $\mathrm{as}^{45}$ :

$$
\nabla \cdot[\varepsilon(r) \nabla \theta(r)]=-4 \pi\left(\rho(r)-\sum_{i=1}^{K} C_{i}^{b u l k} Z_{i}^{2} e_{0}^{2} \frac{\phi(r)}{N_{A} k T}\right),
$$

where $e_{0}$ is the unit charge, $Z_{i}$ is the valency of the ions, $c_{i}^{\text {bulk }}$ is the bulk concentration, $K$ is the types of ions, $T$ is the temperature, $k$ the Boltzmann constant, and $N_{A}$ is the Avogadro constant. For complex systems such as peptides, the above equation is solved numerically. The details of the numerical calculation are given in the Methods section.

$\Delta G_{n p}$ is the cost of cavity formation in the aqueous phase and is estimated $\mathrm{as}^{37}$ :

$$
\Delta G_{n p}=\gamma A+b
$$

where $A$ is the aqueous solvent-accessible surface area of the peptide, $\gamma=0.0278 \mathrm{kcal} / \mathrm{mol} \AA^{2}$, and $b=-1.71$ $\mathrm{kcal} / \mathrm{mol}$. The values of $\gamma$ and $b$ were derived from the partitioning of alkanes between liquid alkane and water. ${ }^{46}$ Thus, the nonpolar interactions between the lipid and the solute molecule are considered implicitly. The values of $\gamma$ and $b$ have been used earlier for calculations on membrane 
proteins and compare favorably with experimental results. $^{28-30}$

\section{Model Construction Polyalanine peptides}

Polyalanine peptides with 20, 25, 28, 30, 35, and 40 alanine residues, were modeled as ideal $\alpha$-helices, with $\psi=$ $-47^{\circ}$ and $\phi=-57^{\circ} .3$ The $\mathrm{N}$ - and C-termini were blocked with acetyl and $N$-methyl groups, respectively. In addition, helices with 20 and 30 residues were modeled with charged ends.

\section{WALP peptides}

WALP peptides were modeled as ideal $\alpha$-helices, ${ }^{3}$ with $\psi=-47^{\circ}$ and $\phi=-57^{\circ}$ using CHARMM $^{38}$ to assign the molecular parameters. Peptides with 19, 20, 25, 30, 35, and 40 residues were constructed with blocked ends.

\section{Melittin}

The melittin peptide structure, $2 \mathrm{MLT},{ }^{47}$ was taken from the Protein Data Bank ${ }^{48}$ (PDB), and the N- and C- termini were charged so as to represent the naturally occurring peptide.

\section{Glycophorin}

The backbone structure of the transmembrane segment of the glycophorin $\mathrm{A}$ monomer, code $1 \mathrm{AFO},{ }^{49}$ residues 73-100, was taken from the PDB. The N-terminus was again blocked with an acetyl group and the C-terminus, with an $N$-methyl group. The side-chains were constructed using the software SCWRL ${ }^{50}$ (version 2.0), which determines the most probable rotameric conformations from a backbone-dependent rotamer library. ${ }^{51}$ Steric clashes are relieved systematically by a combinatorial search of rotamers in an order defined by the rotamer library and interaction energy.

All the above peptides were energy-minimized in a dielectric medium of $\varepsilon=2$ using the CHARMM potential ${ }^{38}$ (version 29a2) with 1000 steps of Steepest Descent followed by 100 steps of Conjugate Gradient and 1000 steps of Newton-Raphson minimization with $1 \mathrm{kcal} / \mathrm{mol}$ harmonic constraints.

\section{Calculation of Solvation Energy}

$\Delta G_{\text {solv }}$ was calculated as a function of $v$ and $\theta$ for the above-modeled peptides according to the thermodynamic cycle shown in Figure 3.

\section{Calculation of $\Delta \boldsymbol{G}_{\text {elec }}$}

$\Delta G_{\text {elec }}$ was evaluated numerically by solving the linear Poisson-Boltzmann equation using a finite difference method with the CHARMM software package (version 29a2). ${ }^{38}$ The salt concentration was set to $0.15 \mathrm{M}$. The electrostatic potentials were calculated using the focusing technique $^{52}$ on a grid, with grid spacing $1.0 \AA, 0.5 \AA$, and $0.25 \AA$. The distance between the grid boundaries and the peptide surface was at least $15 \AA$. The atomic radii were taken as the Born radii. ${ }^{53} \mathrm{~A}$ cardinal b-spline was used for distributing charges over the grid points. The calculations were done at $300 \mathrm{~K}$ and no membrane potential was applied. The electrostatic potentials in the membrane environment and in vacuum $(\varepsilon=1)$ were calculated. The corresponding solvation free energies of the system in the membrane, $\Delta G_{\text {elec }}^{\text {mem }}$, and in vacuum, $\Delta G_{\text {elec }}^{\text {vac }}$, were computed from the above potentials.

\section{Calculation of $\Delta \boldsymbol{G}_{\boldsymbol{n p}}$}

$\Delta G_{n p}$ was calculated using Eq. (4). The solvent-accessible surface area, $A$, was calculated using the CHARMM software $^{38}$ package, with a probe sphere radius of $1.4 \AA$.

\section{Computational requirements}

Each system setup took about $1 \mathrm{~h}$ on four $800 \mathrm{MHz}$ processors in a Linux cluster. Each $\Delta G_{\text {solv }}$ calculation took about $1 \mathrm{~h}$ on a single processor $(2400 \mathrm{MHz}, 2048 \mathrm{MB}$ RAM). The total CPU time required for the calculations in the paper was $\sim 3000 \mathrm{~h}$.

\section{RESULTS AND DISCUSSION}

To understand the energetics of helical peptide orientations in membranes, the solvation free energies of various peptide models are examined in the 5-slab dielectric system described in the Methods section. The results are presented in an order that allow the contributions to the membrane solvation energy to be easily understood. First, we examine the factors determining the location and orientation of a single peptide dipole. Next, we extend the analysis to a simple peptide dipole-containing molecule, $N$-methylacetamide, and to polyalanine helices. In the final sections, comparisons with experiment are made in applications to more complex peptides such as WALPs, glycophorin, and melittin.

\section{Peptide Dipoles}

We begin our analysis by calculating the electrostatic energy of peptide dipoles in membranes. Consider first a simple dipole, consisting of charges $q= \pm 1.0 e$ placed $0.5 \AA$ apart, crossing the 5-slab membrane depicted in Figure 1. The Poisson equation can be solved analytically for this system (see Appendix). We compare 2 orientations of the dipole: $\theta=0^{\circ}$ and $\theta=90^{\circ}$, where $\theta$ is the angle between the dipole axis and the membrane normal. The difference in $\Delta G_{\text {elec }}$ between the $0^{\circ}$ and $90^{\circ}$ orientations is the barrier to flipping, $E_{\text {flip }}$, and gives the preference of one orientation over the other.

Plots of $\Delta G_{\text {elec }}$ versus the membrane insertion distance, $v$, for the 2 orientations of the simple dipole are shown in Figure $4(\mathrm{~A}) . \Delta G_{\text {elec }}$ decreases when traversing from the membrane core to the headgroup region to the aqueous medium, since $\Delta G_{\text {elec }}$ is inversely proportional to $\varepsilon$, which increases in this direction. Further, the difference in $\Delta G_{\text {elec }}$ between the membrane core and headgroup region is approximately 4 times the difference in $\Delta G_{\text {elec }}$ between the headgroup region and water. This difference in $\Delta G_{\text {elec }}$ arises due to the variation of $\varepsilon$ (and hence the potential) in the 3 media and equals $\left(\frac{1}{\varepsilon_{1}}-\frac{1}{\varepsilon_{2}}\right)\left(\frac{1}{\varepsilon_{2}}-\frac{1}{\varepsilon_{3}}\right)^{-1}$, where $\varepsilon_{1}, \varepsilon_{2}$, 
A:

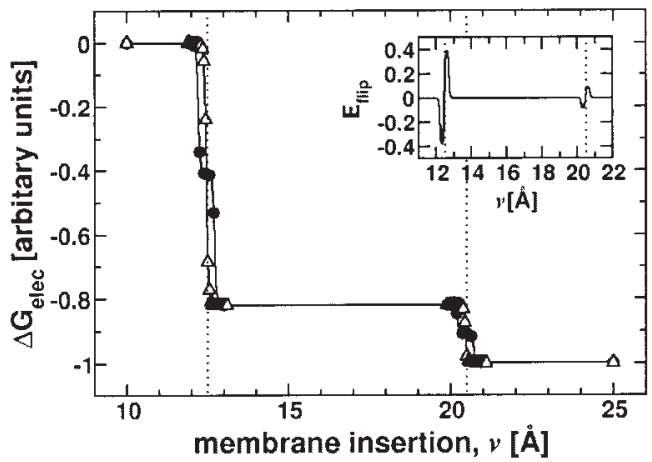

B:

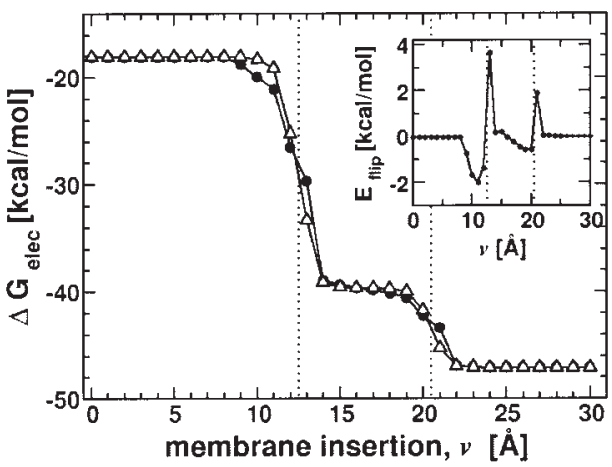

Fig. 4. $\Delta G_{\text {elec }}$ versus membrane insertion, $v$, for (A) dipole (dipole moment $=2.4 \mathrm{D}$ ) calculated by the analytical solution of the Poisson equation, and (B) N-methylacetamide calculated using the model described in the Methods section. Two orientations, $\theta=0^{\circ}(\bullet)$ and $\theta=90^{\circ}$ $(\triangle)$ are considered. The energy of flipping in the membrane, $E_{\text {flip }}$ is given by the difference of $\Delta G_{e l e c}$ of the 2 orientations at a given value of $v$. The two dotted lines depict the boundaries of the headgroup region. $E_{f i p}$ is nonzero only near the boundary between 2 media, giving rise to a preference of one conformation over the other.

and $\varepsilon_{3}$ are the dielectric constants of the membrane core, headgroup, and water, respectively.

In a homogeneous medium, such as the membrane interior, the center of the headgroup region, or the aqueous region, there is no orientational preference of $\Delta G_{\text {elec }}$ and hence no tendency of the dipole to orient itself in a particular direction. In contrast, a difference in $\Delta G_{\text {elec }}$ of the 2 orientations is seen near the dielectric boundaries (i.e., when the dipole traverses from the membrane core to the headgroup region or from the headgroup region to the aqueous layer). Near a dielectric boundary, if the dipole axis is parallel to the membrane normal, then a reaction field countering the charge closest to the boundary builds in the medium with higher polarity, lowering $\Delta G_{\text {elec }}$. In contrast, if the dipole axis is perpendicular to the membrane normal, the reaction fields due to the 2 charges cancel each other, and there is no net lowering of $\Delta G_{\text {elec }}$. Thus, near a boundary between the dipole-containing medium and a higher polarity medium, the orientation with the dipole axis parallel to the membrane normal is favored over the perpendicular orientation. However, this picture reverses once the midpoint of the boundary is reached. On crossing this point, the dipole with $\theta=90^{\circ}$ is completely immersed in the more polar medium and is hence more favorable.

The analysis is now extended to a molecule containing a single peptide bond: $N$-methylacetamide. For this molecule, $\Delta G_{\text {elec }}$ was calculated numerically, as described in the Methods section. Figure 4(B) shows the plot of $\Delta G_{\text {elec }}$ versus $v$ at $\theta=0^{\circ}$ and $\theta=90^{\circ}$. The results are qualitatively similar to the simple dipole model in Figure 4(A). Thus, the energetics of the orientational dependence of $N$-methylacetamide is well described by that of a simple dipole.

\section{Polyalanine Peptides}

The investigation is now extended from a single-peptide group model to polyalanine peptides of varying lengths. Polyalanine peptides have been shown to adopt predominantly helical conformations in lipid bilayers. ${ }^{13}$ Here we present the results of a decomposition of the various factors governing the orientation in a membrane of these simple helices.

\section{Effect of different classes of charged groups on $\Delta \boldsymbol{G}_{\text {elec }}$}

The chemical groups in a helix can be divided into the peptide backbone, the side-chains, and the $\mathrm{N}$ - and $\mathrm{C}$ terminal end groups. The contributions of each of these 3 classes to $\Delta G_{\text {elec }}$ in a 30-mer polyalanine peptide are shown in Figure 5(A and $\mathrm{B})$. Two types of end groups, charged $\left(\mathrm{NH}_{3}^{+}\right.$and $\left.\mathrm{CO}_{2}^{-}\right)$and blocked (acylated N-terminus and $\mathrm{N}$-methylated C-terminus), were also examined.

To calculate the contribution of the above classes to the total $\Delta G_{\text {elec }}$ for the blocked endgroup 30-mer peptide, the partial charges of all other atoms except those constituting the selected groups were set to zero [Fig. 5(A)]. The figure shows that the side-chains contribute very little to the total $\Delta G_{\text {elec }}$ barrier. The blocked peptide end groups make the second lowest contribution. The peptide backbone contributes the most, about 3 times more than the endgroups. Thus, it is indeed the peptide group dipoles that play the major role in orienting the polyalanine helix axis perpendicular to the membrane surface.

The presence of charged termini increases the energy difference between the orientation with $\theta=0^{\circ}$ and $90^{\circ}$. This increase arises from the fact that it is energetically less favorable to bury the charged endgroups than the blocked ones. In contrast, there is little difference in the minimum-energy tilt angle between the blocked and charged peptides [Fig. 5(B)].

\section{Peptide length}

We now further develop the analysis by calculating the solvation energy, $\Delta G_{\text {solv }}$ (i.e., by adding in the nonpolar term, $\Delta G_{n p}$, and by examining the dependence of the minimum-energy tilt angle on peptide length). The minimum-energy tilt angles for polyalanine peptides with 20 , $25,28,30,35$, and 40 residues are plotted in Figure 6(A). The membrane thickness is $41 \AA$, corresponding approximately to a helix of 27 residues. The length of the 20 -mer polyalanine peptide is thus shorter than the bilayer thickness. Consequently, the lowest energy orientation is paral- 
A:
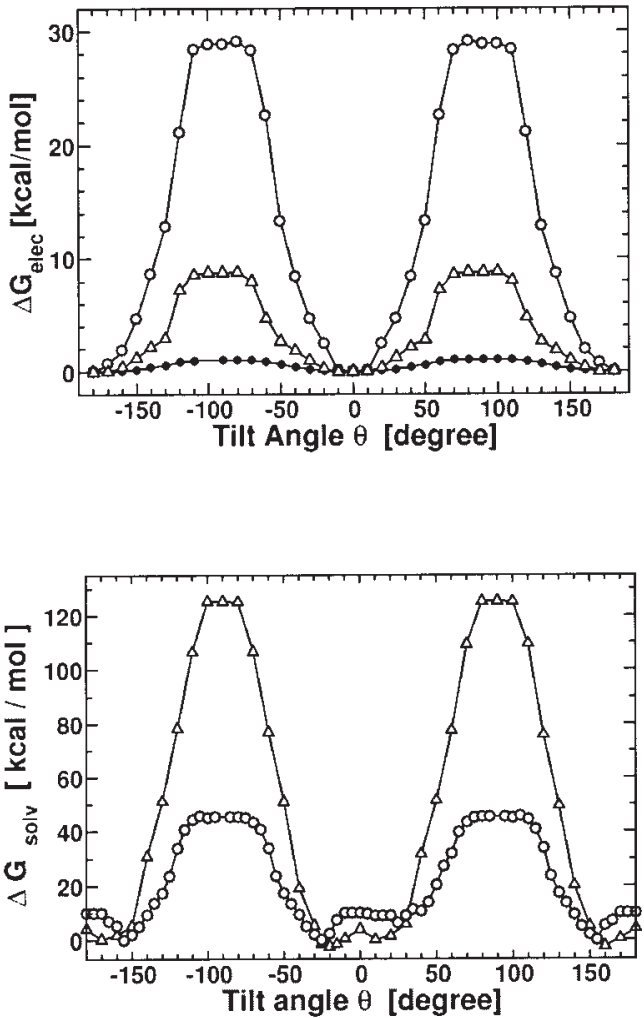

Fig. 5. (A) $\Delta G_{e l e c}$ versus tilt angle, $\theta$, for a 30-mer polyalanine peptide with blocked ends, with partial charges only on the atoms constituting the side-chains $(\bullet), \mathrm{N}$ - and $\mathrm{C}$ - termini $(\triangle)$, or backbone $(\bigcirc)$. The contribution of the backbone atoms to the energy of flipping in a membrane, $E_{f l i p}$, is the largest. The total energy cannot be expressed as a sum of the different contributions due to the interaction terms. (B) $\Delta G_{\text {sorv }}$ versus $\theta$ at $v=0$ for a 30-mer polyalanine peptides with charged ends $(\triangle)$ and blocked ends $(\bigcirc)$. The energies in both (A) and $(B)$ are relative to the lowest energy conformer.

lel to the membrane normal (i.e., $\theta=0^{\circ}$ ), so as to optimize the above-characterized electrostatic energy of the peptide dipoles.

For the peptides longer than the bilayer width, the cost of cavity formation in the aqueous layer $\left(\Delta G_{n p}\right)$ is large enough to cause tilting of the helices so as to decrease the surface area exposed to the aqueous medium. Thus, for the 28-mer polyalanine peptide, which is longer than the membrane thickness, a nonzero tilt is observed. A further increase in tilt angle is observed with increasing number of residues. The minimum-energy tilt angle increases roughly linearly with the peptide length, as is evident in Figure 6(A). Approximating the $\alpha$-helix as a cylinder of length, $l$ and radius $r$, the exposed surface area at $\theta=0^{\circ}$ is given by $A=2 \pi r(l-d)$, where $d$ is the width of the bilayer. Since $\Delta G_{n p}$ is proportional to the exposed surface area, the contribution of $\Delta G_{n p}$ to the total $\Delta G_{\text {solv }}$ increases approximately linearly.

To estimate the dynamic fluctuation of the tilt angle, a harmonic function was fitted to the solvation free energy profiles in the low-energy region. The resulting thermal fluctuations of the tilt angles, calculated from the fitted harmonic potentials, are also shown in Figure 6(A) as bars. The fluctuations of the peptides decrease with increasing
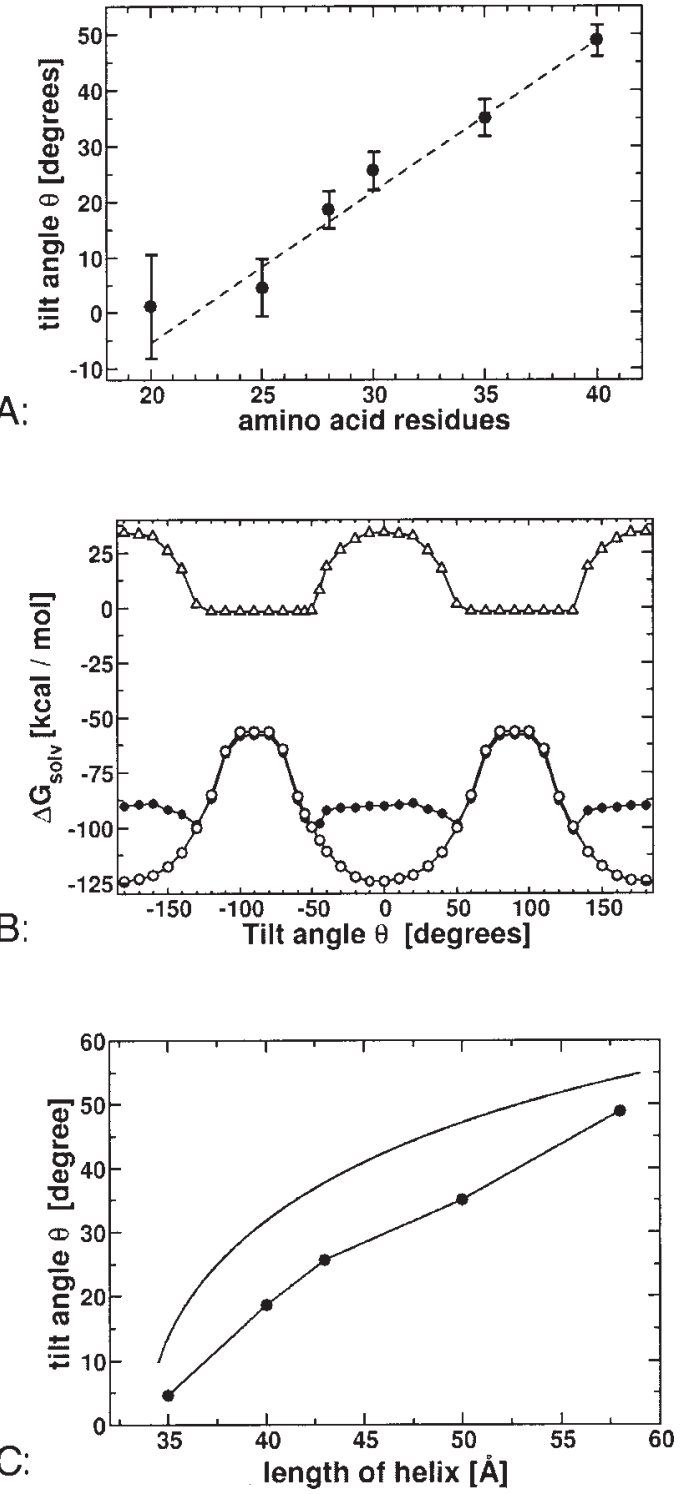

Fig. 6. Calculated orientations of polyalanine peptides in a membrane environment. (A) Minimum-energy tilt angles versus number of residues for polyalanine peptides at $v=0 \AA$. The thermal fluctuations given by the bars were calculated by fitting a harmonic potential to the energy profile of each peptide. (B) $\Delta G_{\text {solv }}$ versus $\theta$ for a 40-mer with blocked ends at $v=0$ $\AA$. The electrostatic, $\Delta G_{\text {elec }}(\bigcirc)$, and nonpolar, $\Delta G_{n p}(\triangle)$, components of the total $\Delta G_{\text {solv }}(\bullet)$ are also plotted. (C) The minimum-energy $\theta(\bullet)$ and that predicted by a geometric model ( - in which the tilt angle adopted is the smallest such that the peptide just spans the bilayer (see text).

helix length. Large fluctuations are possible in shorter peptides such as the 20-mer, since for $v=0 \AA, \Delta G_{\text {elec }}$ is relatively broad and the peptides are never exposed to the aqueous layer for any value of $\theta$. In contrast, fluctuation of the helix axis for the longer peptides leads to stronger variations in $\Delta G_{n p}$ and $\Delta G_{\text {elec }}$. Thus, the longer peptides are locked in steeper potentials. The above aspects of the free energy profiles are evident in Figure 6(B), in which $\Delta G_{\text {solv }}, \Delta G_{\text {elec }}$, and $\Delta G_{n p}$ are plotted versus $\theta$ for the 40 -mer polyalanine peptide. This decomposition also illustrates 


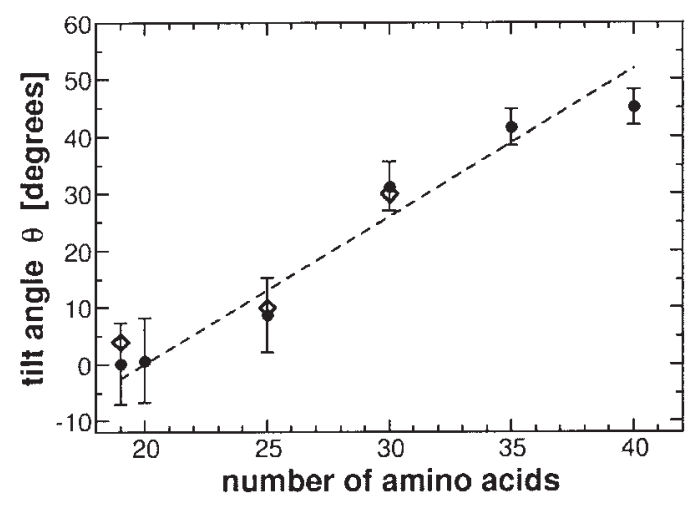

Fig. 7. Minimum-energy tilt angle as a function of residue length for WALP peptides at $v=0 \AA$. The theoretically determined tilt angles $(\bullet)$ are represented, and the bars correspond to the thermal fluctuations, calculated by fitting a harmonic potential to the energy profile. The experimentally determined tilt angles ${ }^{16,18}$ are represented by $(\diamond)$. The main contribution to the nonzero tilt angles arises from $\Delta G_{n p}$. Since $\Delta G_{n p}$ increases linearly with the exposed surface area, the graph is approximately linear.

how the tilting of the longer polyalanine peptides is due mainly to the contribution of $\Delta G_{n p}$.

It has been proposed that, for short peptides, bilayer thinning occurs to compensate for the hydrophobic mismatch between the membrane thickness and peptide length. ${ }^{15}$ Reducing the membrane core thickness from 25 $\AA$ to $20 \AA$ did not lead to a change in the tilt angle for the 20-mer polyalanine peptide. Further, to test whether the dielectric constant of the nonpolar membrane core affects the results, a control calculation, increasing the dielectric constant of the core region to $\varepsilon=4$, was carried out for the 40-mer. Again, the tilt angle did not change, remaining at $40^{\circ}$.

The results in Figure 6(A) suggest a simple geometric model in which the tilt angle adopted by a helix is the smallest possible, while ensuring that the length of the helix can be fully incorporated within the bilayer. According to this model, $d=l \cos \theta$, where $d$ is the width of the bilayer and $l$ is the length of the helix. However, as shown in Figure 6(C), this model overestimates the tilt angles. Thus the balance between $\Delta G_{\text {elec }}$ and $\Delta G_{n p}$ is not well represented by the simple geometric model.

\section{WALP Peptides}

The WALP peptides are of particular interest, as experimental $\mathrm{NMR}^{16}$ and spectroscopic ${ }^{18}$ data exist on their tilt angles. Therefore, calculations similar to those discussed above for the polyalanines were performed for WALP peptides with varying numbers of amino-acid residues. The resulting tilt angles and corresponding fluctuations are plotted in Figure 7.

As for the polyalanine helices, the tilt angle for the 19-mer and 20-mer WALP peptide is $0^{\circ}$, with the peptides orienting perpendicular to the membrane plane. The tilt angle for the longer peptides again deviates from zero, increasing with the number of residues. The tilt angles are found to be in good agreement with experiment.

A further point of interest is that in the experimental determination of the tilt angle of the 19-mer WALP peptide, it was suggested that this peptide has a nonzero tilt angle but is nevertheless oriented roughly perpendicularly to the membrane plane. ${ }^{16}$ The present analysis suggests that the relatively large fluctuations of the 19mer WALP peptide tilt angle may have led to this observation.

To test whether the bilayer headgroup region contributes significantly to the final tilt angle and corresponding fluctuations of a peptide, a control calculation of the orientation of a 20-mer WALP peptide was carried out with a 3-slab membrane model as used in previous studies. $^{28}$ Due to the absence of a headgroup region in the bilayer, the tryptophan residues are unable to anchor the peptide to the headgroup region, and the peptide was seen to tilt appreciably: The tilt angle calculated was $21^{\circ}$, with fluctuation of $\sim 4^{\circ}$. These results are in contrast to the calculations with the 5-slab model and also with the corresponding experimental results. Hence, including headgroup energetics is necessary for calculating membranepeptide orientations.

\section{Melittin}

In the final Results section, the model is applied to 2 extensively studied heteropolymeric membrane helices: melittin and glycophorin. The membrane orientation of melittin has been a topic of much debate, especially as the various mechanisms proposed for melittin-mediated cell lysis depend on its membrane orientation., ${ }^{9,21}$ Previous studies have indicated both transmembrane and surfacealigned orientations depending on the experimental conditions. ${ }^{9,54,55}$ At low concentrations in phosphatidylcholine bilayers, the predominant orientation of monomeric melittin determined by X-ray scattering was found to be surface aligned with the helical axis at the depth of the glycerol groups. The amphipathic nature of melittin makes it favorable for it to interact with both the dielectric boundary of the polar aqueous layer and the nonpolar membrane core.

$\Delta G_{\text {solv }}$ was calculated for melittin orientations corresponding to $-180^{\circ} \leq \theta \leq 180^{\circ}$ and $0 \AA \leq v \leq 25 \AA$. In a contour plot of $\Delta G_{\text {solv }}$ versus $\theta$ and $v$ (Fig. 8), the lowest energy orientation is labeled as Region I (lying between $14 \AA \leq v \leq 19 \AA$ and $70^{\circ} \leq \theta \leq 90^{\circ}$ ). This region corresponds to a surface-aligned orientation with the helix axis perpendicular to the membrane normal, consistent with previous theoretical studies. ${ }^{21}$

In the crystal structure of melittin, a kink is formed in the helix backbone due to a proline residue, giving rise to a short C-terminal hydrophobic tail. ${ }^{47}$ Due to this kink, the orientation corresponding to Region I allows the Cterminal tail to interact with the core region of the membrane and is energetically favorable. In contrast, in Region II (i.e., $-90^{\circ} \leq \theta \leq-70^{\circ}$ ), the nonpolar tail interacts with the aqueous layer, increasing $\Delta G_{\text {solv }}$ substantially and making it highly unfavorable.

\section{Glycophorin Monomer}

In Figure 9, the solvation energy, $\Delta G_{\text {solv }}$, of glycophorin is plotted as a function of $\theta$ at $v=0 \AA$. The orientation with 

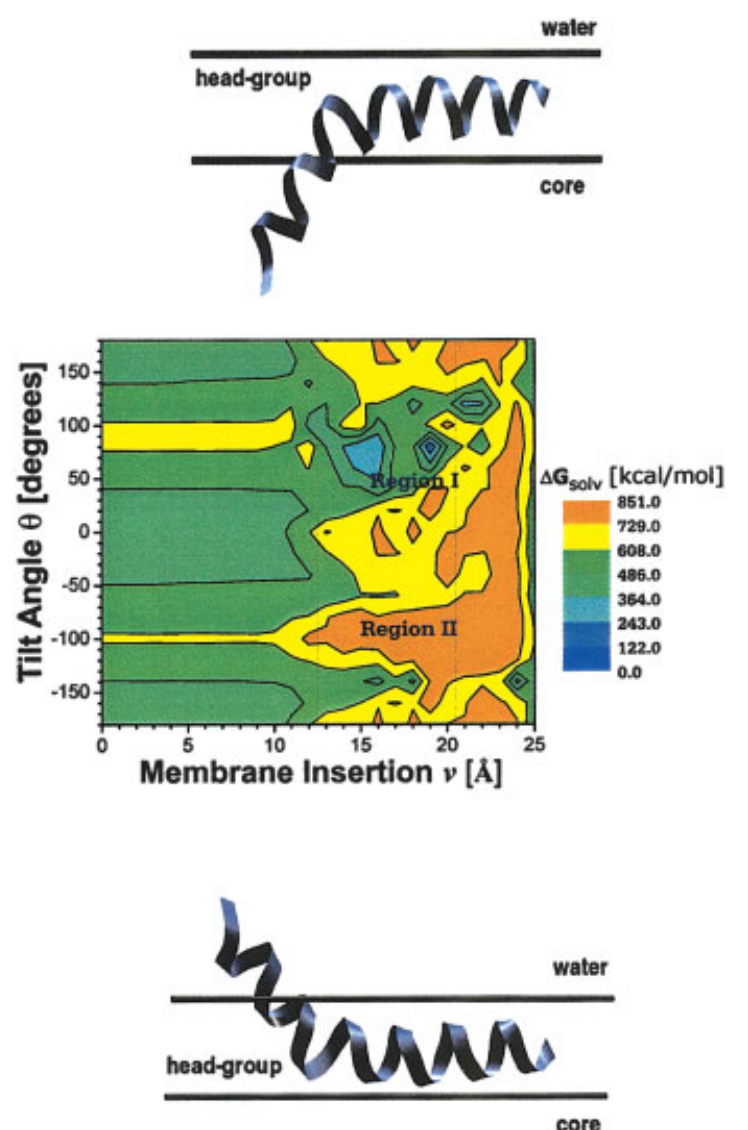

Fig. 8. Solvation energy, $\Delta G_{\text {solv }}$, as a function of tilt angle, $\theta$, and membrane insertion, $v$, for melittin monomers in a membrane environment. The most favorable orientation of melittin is region I, corresponding to a surface-aligned orientation, with the $\mathrm{C}$-terminal tail interacting with the membrane core (top). Region II also corresponds to a surface-aligned orientation but with the C-terminal tail interacting with the water (bottom) and is energetically highly unfavorable. The lowest energy conformers cluster around $80^{\circ} \leq \theta \leq 90^{\circ}$ and $18 \AA \leq v \leq 19 \AA$, and all energies are relative to the lowest energy conformer. The headgroup region extends between $12.5 \AA$ and $20.5 \AA$.

the lowest energy is at $\theta=15^{\circ}$, with thermal fluctuations of $\sim 5^{\circ}$. The value is consistent with the experimental tilt angle of $\sim 20^{\circ}$ determined by solution $\mathrm{NMR}^{49}$ and Fourier transform infrared (FTIR) spectroscopy ${ }^{12}$ of glycophorin monomers. The nonzero tilt angle stems in this case mainly from the $\Delta G_{\text {elec }}$ contribution. The transmembrane segment of glycophorin consists of several long-chain and aromatic side-chains and is thus more nonpolar than peptides containing many alanine residues. Tilting of the helix incorporates more of these side-chains into the nonpolar membrane core region, while allowing the flanking polar residues to interact with the headgroup region. This gives rise to a nonzero tilt angle in glycophorin, in contrast to the zero tilt angle in WALP peptides of similar length.

\section{CONCLUSIONS}

In the work presented here, the membrane orientations of single $\alpha$-helices have been quantitatively examined using all-atom models of the helices embedded in a 5-slab

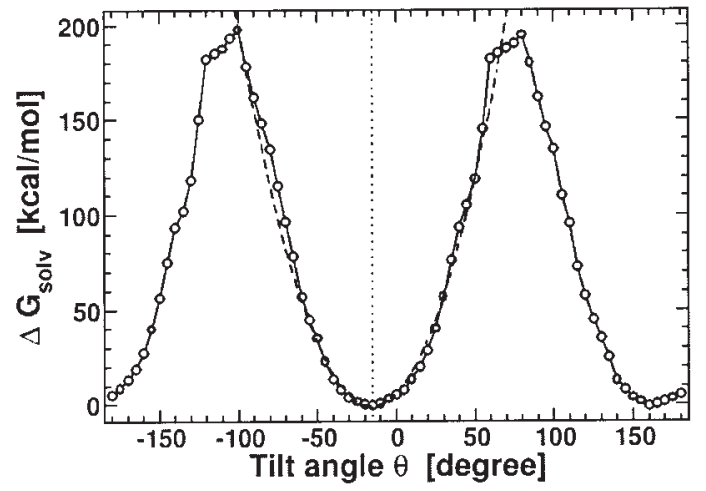

Fig. 9. Solvation energy, $\Delta G_{\text {solv }}$, versus tilt angle, $\theta$, for the glycophorin monomer in a membrane. $\Delta G_{\text {solv }}$ was calculated by the thermodynamic cycle shown in Figure 3 . The lowest energy orientation is at $\theta=15^{\circ}$ and $v=0 \AA$. The energies are relative to the lowest energy conformer. A harmonic potential (dotted curve) is fitted on the energy profile between $\theta=25^{\circ}$ and $\theta=5^{\circ}$. The resulting tilt angle is $14^{\circ}$, with thermal fluctuations of $\sim 5^{\circ}$. The experimentally determined tilt angle is $20^{\circ} .{ }^{26}$

continuum dielectric environment representing the membrane and associated aqueous phase.

The model has allowed the energetic factors determining the helix orientations to be quantitatively assessed. The approach taken was to start from considerations of a simple dipole in a membrane and then to build in additional effects as modeled by an all-atom peptide force field and burial of accessible surface area. The peptide dipoles are shown to favor the orientation perpendicular to the membrane plane via a solvent reaction field effect that is significant only for groups that approach the dielectric boundaries of the system. This reaction field is shown to be responsible for determining the membrane orientation of blocked polyalanine helices. Another important effect is solvent cavity formation that tends to minimize the surface area accessible to water. According to the model, the cavity term plays an important role in influencing the steepness of the tilt angle potential, such that the longer peptides undergo smaller tilt angle fluctuations than the shorter ones. The combination of solvation terms also allows the rationalization of the length dependence of the peptide tilt angle on length. Furthermore, the presence of a separate dielectric zone for the headgroup region is found to be important in determining the helix orientational properties.

Importantly, the model is found to correctly reproduce the helix tilt angles of peptides for which the tilt angles have been determined experimentally. These comprise the length-dependent helix tilt angles of the WALP peptides, together with the membrane orientations of melittin and glycophorin. The comparison with experiment provides evidence that the model quantitatively captures the main elements of the orientation-dependent membrane-helix solvation energy. The present model is potentially useful in 2 domains. First, the model can provide starting geometries for detailed MD simulations of helices in membranes, in which all helix, membrane, and water atoms are explicitly represented..$^{20-27}$ These simulations, although time-consuming, can provide a detailed picture of the 
helix-membrane system and include effects such as internal peptide flexibility and explicit peptide-lipid interactions.

Second, the model may provide a stepping stone toward obtaining a theoretical framework for determining the structures of the many important proteins that contain helical transmembrane domains and that are difficult to crystallize. To do this, it will be necessary to search relative helix translational and rotational angles in helix oligomers and to include backbone and side-chain flexibility. As a first step in this direction, calculations are in progress on the membrane association of helices into dimers.

\section{REFERENCES}

1. White SH, Wimley WC. Membrane protein folding and stability: physical principles. Annu Rev Biophys Biomol Struct 1999;28:319336.

2. Yeagle PL, Lee AG. Membrane protein structure. Biochim Biophys Acta 2002;1565:143.

3. Branden C, Tooze J. Introduction to protein structure. New York: Garland; 1998.

4. Ubarretxena-Belandia I, Engelman DM. Helical membrane proteins: diversity of functions in the context of simple architecture. Curr Opin Struct Biol 2001;11:370-376.

5. White SH, Wimley WC. Hydrophobic interactions of peptides with membrane interfaces. Biochim Biophys Acta 1998;1376:339-352.

6. Popot JL, Engelman DM. Membrane protein folding and oligomerization - the 2-stage model. Biochemistry 1990;29:4031-4037.

7. Popot JL, Engelman DM. Helical membrane protein folding, stability and evolution. Annu Rev Biochem 2000;69:881-922.

8. Torres J, Kukol A, Arkin IT. Use of a single glycine residue to determine the tilt and orientation of a transmembrane helix: a new structural label for infrared spectroscopy. Biophys J 1999;79: $3139-3143$.

9. Hristova K, Dempsey CE, White SH. Structure, location, and lipid perturbations of melittin at the membrane interface. Biophys $\mathrm{J}$ 2001;80:801-811.

10. Watts A, Burnett IJ, Glaubitz C, Grobner G, Middleton DA, Spooner PJR, Watts JA, Williamson PTF. Membrane protein structure determination by solid state NMR. Nat Prod Rep 1999;16:419-423.

11. Mesleh MF, Lee S, Veglia G, Thiriot DS, Marassi FM, Opella SJ. Dipolar waves map the structure and topology of helices in membrane proteins. J Am Chem Soc 2003;125:8928-8935.

12. Arkin IT, Mackenzie KR, Bruenger AT. Site-directed dichroism as a method for obtaining rotational and orientational constraints for oriented polymers. J Am Chem Soc 1997;119:8973-8980.

13. Bechinger B. Membrane insertion and orientation of polyalanine peptides: a $15 \mathrm{n}$ solid state NMR spectroscopy investigation. Biophys J 2001;81:2251-2256.

14. Braun P, Heijne GH. The aromatic residues Trp and Phe have different effects on the positioning of a transmembrane helix in the microsomal membrane. Biochemistry 1999;38:9778-9782.

15. Demmers JAA, van Duijn E, Haverkamp J, Greathouse DV, Koeppe RE II, Heck AJR, Greathouse JA. Interfacial positioning and stability of transmembrane peptides in lipid bilayers studies by combining hydrogen/deuterium exchange and mass spectroscopy. J Biol Chem 2001;276:34501-34508.

16. van der Wel PCA, Strandberg E, Killian JA, Koeppe RE. Geometry and intrinsic tilt of a tryptophan-anchored transmembrane $\alpha$-helix determined by $2 \mathrm{H}$ NMR. Biophys J 2002;83:1479-1488.

17. Weiss TM, van der Wel PCA, Killian JA, Koeppe RE II, Huang HW. Hydrophobic mismatch between helices and lipid bilayers. Biophys J 2003;84:379-385.

18. de Planque MRR, Goormaghtigh E, Greathouse DV, Koeppe RE II, Kruijtzer JAW, Liskamp RMM, de Kruiff B, Killian JA. Sensitivity of single membrane spanning $\alpha$-helical peptides to hydrophobic mismatch with a lipid bilayer: effects on backbone structure, orientation and extent of membrane incorporation. Biochemistry 2001;40:5000-5010.

19. Sharpe S, Barber KR, Grant CWM, Goodyear D, Morrow MR.
Organisation of model helical peptides in lipid bilayers: insight into the behaviour of single-span protein transmembrane domains. Biophys J 2002;83:345-358.

20. Mihailescu D, Smith JC. Atomic detail peptide-membrane interactions: molecular dynamics of gramicidine $\mathrm{S}$ in a DMPC bilayer. Biophys J 2000;79:1718-1730.

21. Bernéche S, Nina M, Roux B. Molecular dynamics simulation of melittin in a dimyristoyl phosphatidylcholine bilayer membrane. Biophys J 1998;75:1603-1618.

22. Tieleman DP, Breed J, Berendsen HJC, Sansom MSP. Alamethicin channels in a membrane: molecular dynamics simulations. Faraday Disc 1998;111:209-225.

23. Tieleman DP, Berendsen HJC, Sansom MSP. Surface binding of alamethicin stabilizes its helical structure molecular dynamics simulations. Biophys J 1999;76:3186-3191.

24. Woolf TB, Roux B. Structure, energetics and dynamics of lipidprotein interactions: a molecular dynamics study of gramicidine a channel in a dmpc bilayer. Proteins 1996;24:92-114.

25. Capener CE, Shrivastava IH, Ranatunga KM, Forrest LR, Smith GR, Sansom MSP. Homology modeling and molecular dynamics simulation studies of an inward rectifier potassium channel. Biophys J 2000;78:2929-2942.

26. Kukol A, Adams PD, Rice LM, Brunger AT, Arkin IT. Experimentally based orientational refinement of membrane protein models: a structure for the Influenza A M2 $\mathrm{h}^{+}$channel. $\mathrm{J}$ Mol Biol 1999;286:951-962.

27. Forrest L, Kukol A, Arkin I, Tielman A, Sansom M. Exploring the models of Influenza M2 channel-MD simulations in a phospholipid bilayer. Biophys J 2000;78:79-92.

28. Kessel A, Cafiso DS, Ben-Tal N. Continuum solvent model calculations of alamethicinmembrane interactions: thermodynamic aspects. Biophys J 2000;78:571-583.

29. Ben-Tal N, Ben-Shaul A, Nicholls A, Honig B. Free-energy determinants of alpha-helix insertion into bilayers. Biophys $\mathrm{J}$ 1996;70:1803-1812.

30. Murray D, Hermida-Matsumoto L, Buser CA, Tsang J, Sigal CT, Ben-Tal N. Electrostatics and the membrane association of Src: theory and experiment. Biochemistry 1998;37:2145-2159.

31. Diraviyam K, Stahelin RV, Cho W, Murray D. Computer modeling of the membrane interaction of FYVE domains. J Mol Biol 2003;328:721-736.

32. Still WC, Tempczyk A, Hawley RC, Hendrickson T. Semianalytical treatment of solvation for molecular mechanics and dynamics. J Am Chem Soc 1990;112:6127-6129.

33. Im W, Feig M CLB III. An implicit membrane generalized Born theory for the study of structure, stability, and interactions of membrane proteins. Biophys J 2003;85:2900-2918.

34. Spassov VZ, Yan L, Szalma S. Introducing an implicit membrane in generalized Born/solvent accessibility continuum solvent models. J Phys Chem B 2002;106:8726-8738.

35. Grossfield A, Sachs J, Woolf TB. Dipole lattic membrane model for protein calculation. Proteins 2000;41:211-233.

36. Lazardis T. Effective energy function for proteins in lipid membranes. Proteins 2003;52:176-192.

37. Leach AR. Molecular modelling: principles and applications. Harlow: Pearson Education Limited, EMA; 2001.

38. Brooks BR, Bruccoleri RE, Olafson BD, States DJ, Swaminathan S, Karplus M. CHARMM: a program for macromolecular energy, minimization, and dynamics calculation. J Comp Chem 1983;4: 187-217.

39. Stern HA, Feller SE. Calculation of dielectric permittivity profile for a nonuniform system: application to a lipid bilayer simulation. J Chem Phys 2003;118:3401-3411.

40. Zhou F, Schulten K. Molecular dynamics study of a membranewater interface. J Phys Chem 1995;99:2194-2207.

41. Coster HGL, Smith JR. The molecular organization of bimolecular lipid membranes: a study of low frequency Maxwell-Wagner impedance dispersion. Biochim Biophys Acta 1974;373:151-164.

42. Cseh R, Benz R. Interaction of phloretin with lipid monolayers: relationship between structural changes and dipole potential change. Biophys J 1999;77:1477-1488.

43. Cevc G,Watts A, Marsh D. Titration of the phase transition of phosphatidylserine bilayer membranes. effects of $\mathrm{pH}$, surface electrostatics, ion binding and head group hydration. Biochemistry 1981;20:4955-4965.

44. Lelkes PI, Miller IR. Perturbations of membrane structure by 
optical probes: location and structural sensitivity of merocyanine 540 bound to phospholipid membranes. J Mol Biol 1980;52:1-15.

45. Jackson J. Classical electrodynamics. New York: Wiley; 1998.

46. Sitkoff D, Ben-Tal N, Honig B. Calculation of alkane to water solvation free energies using continuum solvent models. J Phys Chem 1996;100:2744-2752.

47. Terwilliger TC, Eisenberg D. The structure of melittin. I. Structure determination and partial refinement. J Biol Chem 1982; 257:6010-6015.

48. Berman HM,Westbrook J, Feng Z, Gilliard G, Bhat TN, Weissig $\mathrm{H}$, Shindyalov IN, Bourne PE. The protein data bank. Nucleic Acids Res 2000;28:235-242.

49. MacKenzie KR, Prestegard JH, Engelman DM. A transmembrane helix dimer: structure and implications. Science 1997;276:131133.

50. Bower M, Cohen FE, Dunbrack RL Jr. Side-chain prediction from a backbone dependent rotamer library: a new tool for homology modeling. J Mol Biol 1997;267:1268-1282.

51. Dunbrack RL Jr, Karplus M. Backbone-dependent rotamer library for proteins: application to side-chain prediction. J Mol Biol 1993;230:543-574.

52. Im W, Beglov D, Roux B. Continuum solvation model: computation of electrostatic forces from numerical solutions to the PoissonBoltzmann equation. Comp Phys Commun 1998;109:1-17.

53. Nina M, Beglov D, Roux B. Atomic Born radii for continuum electrostatic calculations based on molecular dynamics. J Phys Chem 1997;101:5239-5248.

54. Frey S, Tamm LK. Orientation of melittin in phospholipid bilayers: a polarised ATRI study. Biophys J 1991;60:922-930.

55. Bradshaw JP, Dempsey CE, Watts A. A combined x-ray neutron diffraction study of selectively deuterated melittin in phospholipid bilayers: effect of pH. Mol Membr Biol 1994;11:79-86.

\section{APPENDIX}

\section{Analytical Solution of Poisson Equation for a Dipole in a Membrane}

To obtain the results in Figure 4(A), the Poisson equation was solved analytically for a dipole in the 5-slab continuum model. The Poisson equation is

$$
\nabla \cdot[\varepsilon(r) \nabla \phi(r)]=-4 \pi \rho(r)
$$

where, $\phi$ is the potential within a medium of uniform dielectric constant $\varepsilon$, and the charge distribution is $\rho$. We consider the distance between the 2 dielectric boundaries to be large enough for the dipole charges to experience only 1 dielectric boundary at a time. This approximation was validated by a numerical solution in which a dipole of the same dipole strength traversed the 5-slab membrane model. The numerical solution showed that toward the center of the headgroup region, $\Delta G_{\text {elec }}$ was constant, indicating a homogeneous field, and that at either dielectric boundary the change of $\Delta G_{\text {elec }}$ was independent of the other dielectric boundary.

The total electrostatic potential $\Phi$ can be written as

$$
\phi=\phi_{q_{a}}+\phi_{q_{b}}
$$

where $\phi_{q_{a}}$ and $\phi_{q_{b}}$ are the potentials due to charge $q_{a}$ and $q_{b}$, respectively. For a dipole, $q_{a}=-q_{b}$. The potential, $\phi_{q}$ at point $\mathrm{P}$, in cylindrical coordinates $(\rho, \phi, z)$, due to a single charge $q$ placed a distance $d$ away from a dielectric boundary between media with dielectric constants $\varepsilon_{1}$ and $\varepsilon_{2}$ is (see Fig. 10) ${ }^{45}$

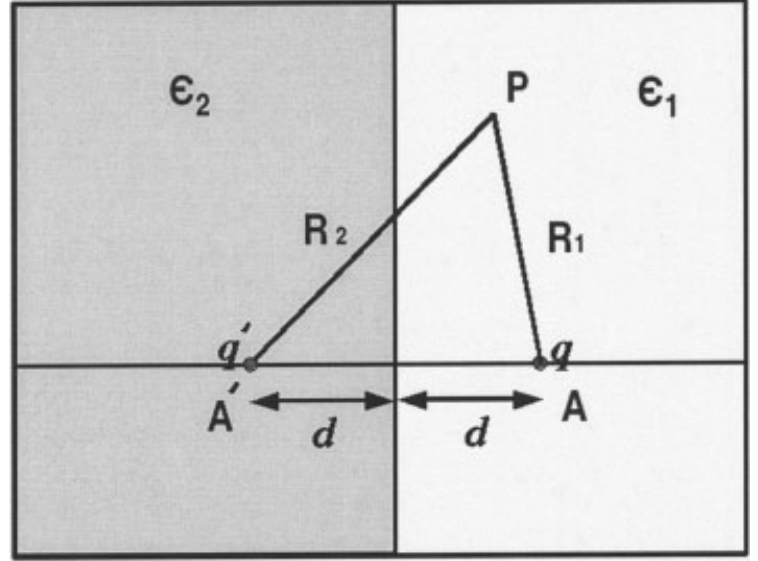

Fig. 10. A single charge, $q$, and its mirror charge, $q^{\prime}$, each a distance, $d$, away from the boundary between media with dielectric constants $\varepsilon_{1}$ and $\varepsilon_{2}$. The potential $\phi$ is calculated at point $P$, which is at distance $R_{1}$ from $q$.

$$
\begin{aligned}
\phi=\frac{1}{4 \pi \varepsilon_{1}}\left(\frac{q}{R_{1}}+\frac{q^{\prime}}{R_{2}}\right) & z>0 \\
\phi=\frac{1}{4 \pi \varepsilon_{2}} \frac{q^{\prime \prime}}{R_{1}} & z<0,
\end{aligned}
$$

where $R_{1}=\sqrt{\rho^{2}+(d-z)^{2}}$ and $R_{2}=\sqrt{\rho^{2}+(d+z)^{2}}$. The image charges $q^{\prime}$ and $q^{\prime \prime}$ are chosen to satisfy the boundary conditions for the electric field $E$, that is,

$$
\lim _{z \rightarrow 0^{+}}\left\{\begin{array}{c}
\varepsilon_{1} E_{z} \\
E_{\rho}
\end{array}\right\}=\lim _{z \rightarrow 0^{-}}\left\{\begin{array}{c}
\varepsilon_{2} E_{z} \\
E_{\rho}
\end{array}\right\} .
$$

Using $E=\nabla \phi_{q}$ and Eq. (7) yields

$$
\begin{aligned}
& q^{\prime}=\frac{\varepsilon_{1}-\varepsilon_{2}}{\varepsilon_{2}+\varepsilon_{1}} q \\
& q^{\prime \prime}=\frac{2 \varepsilon_{2}}{\varepsilon_{2}+\varepsilon_{1}} q .
\end{aligned}
$$

The electrostatic field energy of the dipole is then given by

$$
\begin{gathered}
W=\int{ }_{V} \varepsilon(\mathrm{r}) E^{2} d V \\
=\int_{V} \varepsilon(\mathrm{r})[\underbrace{\left(\nabla \phi_{a}\right)^{2}+\left(\nabla \phi_{b}\right)^{2}}_{\text {self-energy }}+\underbrace{\nabla \phi_{a} \cdot \nabla \phi_{b}}_{\text {interaction energy }}] d V,
\end{gathered}
$$

where $\phi_{a}$ and $\phi_{b}$ are obtained from Eq. (7). Problems arise from the divergent self-energy terms when integrating Eq. (11). These difficulties were avoided by integrating on a plane parallel to the dipole axis, thus excluding the charges. This procedure corresponds to a remapping of the energy density of the electric field from 3 to 2 dimensions. 